\title{
L'académie royale des sciences et les grandes commissions d'enquête et d'expertise à la fin de l'ancien régime
}

\section{Pascale Mafarette-Dayries}

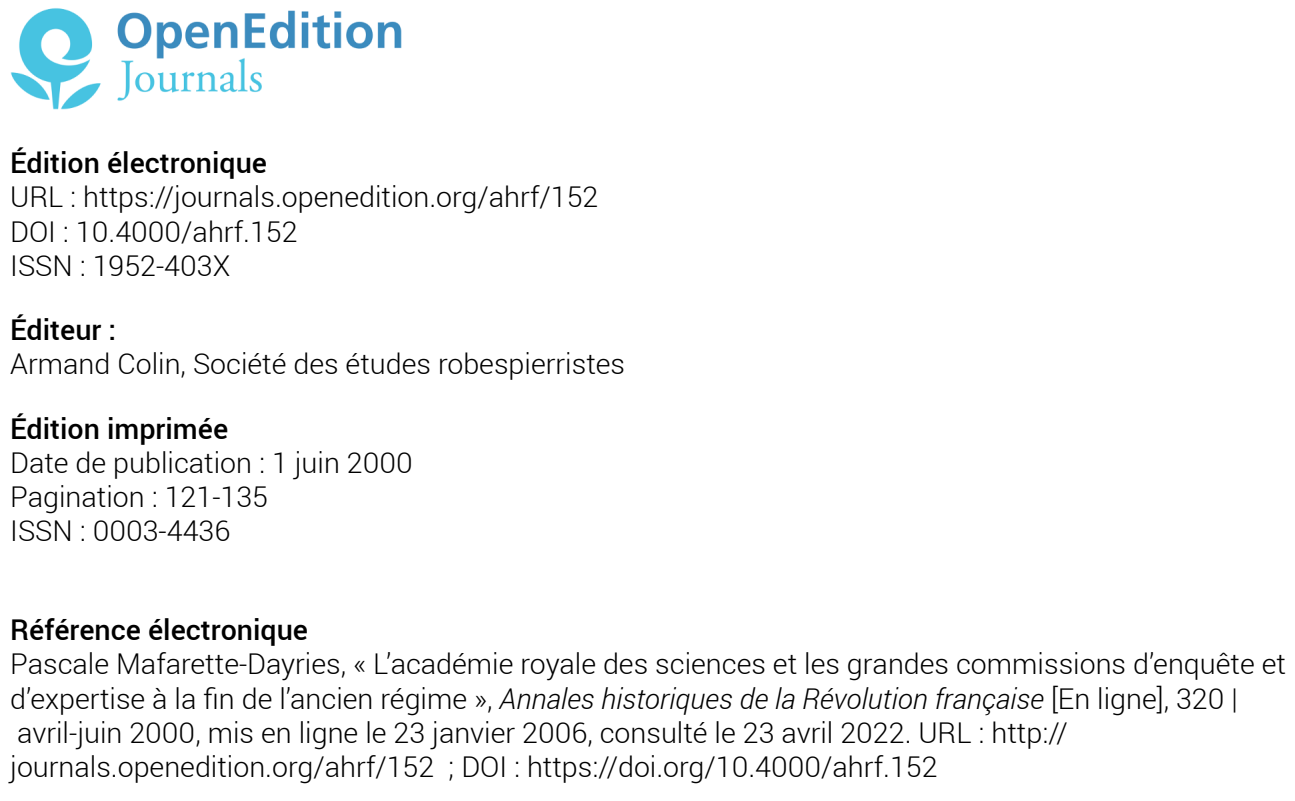

Ce document a été généré automatiquement le 23 avril 2022.

Tous droits réservés 


\title{
L'académie royale des sciences et les grandes commissions d'enquête et d'expertise à la fin de l'ancien régime
}

\author{
Pascale Mafarette-Dayries
}

1 Création de Colbert, l'Académie royale des sciences est intimement liée à l'État dont elle sert les visées utilitaires. Établissement de cartes et de plans, examen de mémoires pour la délivrance de privilèges, l'Académie est tout à la fois expert, juge, enquêteur et auteur de projets scientifiques.

2 À l'aube de la Révolution, son rôle s'est considérablement élargi, dépassant le cadre de son domaine de compétence initial. L'étude des commissions académiques, qui sont un des moteurs essentiels de la vie de la Compagnie, révèle sous une apparente transparence et sérénité, tout un monde de non-dits, d'alliances et de réseaux, de combats scientifiques et de préséance mais aussi de relations au pouvoir politique.

3 Ces commissions, hétérogènes par leur constitution, leur objet et leur mode opératoire, doivent se transformer pour faire face aux besoins de la recherche, répondre aux demandes croissantes d'un public qui amalgame dans un même enthousiasme les travaux scientifiques et les spectacles des charlatans et satisfaire enfin aux nouvelles exigences d'un État soucieux du bien-être de ses sujets et du développement économique du pays. La multiplication des échanges entre le monde savant et des membres du gouvernement et de l'administration durant les dernières années de l'Ancien Régime en est l'illustration'.

4 La composition et le mode de fonctionnement des commissions académiques permettent de mesurer l'évolution des mentalités au sein du corps et l'intensité des échanges avec l'appareil étatique. De grandes commissions, associées aux visées utilitaires du ministre de la Maison du Roi, le baron de Breteuil, contribuent au développement de sa politique et participent à de véritables missions de service public. 
Elles permettent aussi aux savants de s'immiscer dans le domaine du politique et d'intervenir au niveau décisionnel.

5 Parmi les commissions de cinq membres et plus qui seront créées entre 1780 et 1789 , trois d'entre elles éclairent les glissements qui s'opèrent. La commission des ballons, qui marque la difficile mise en place d'une nouvelle organisation du travail scientifique et annonce la nécessaire réforme de l'Académie en 1785, celle sur le Magnétisme animal qui affirme ouvertement l'intrusion du politique dans les travaux académiques et enfin celle sur les hôpitaux qui montre la capacité de l'Académie à répondre aux préoccupations de service public du gouvernement auquel elle apporte sa caution et sa crédibilité.

Les commissions académiques...

6 Parmi les tâches confiées à l'Académie des sciences ${ }^{2}$, il y avait l'examen des divers travaux de particuliers présentés sous forme de mémoires, auxquels s'ajoutaient les recherches des académiciens eux-mêmes ainsi que celles de savants étrangers qui sollicitaient l'avis de leurs confrères. Parallèlement, l'État requérait le concours de l'Académie pour l'exécution de différents travaux qui s'effectuaient parfois aux cours de grandes expéditions. L'augmentation constante des mémoires soumis à l'Académie ainsi que la diversité des solliciteurs suggèrent bien la prééminence croissante de ce corps dans le monde des sciences et des arts appliqués.

7 Toutefois, dans une société où chaque corps défend jalousement ses privilèges et prérogatives, l'Académie était tenue de respecter certaines contraintes de forme et de fond. Elle ne pouvait se saisir elle-même d'un mémoire ou traiter de points ne concernant pas directement l'objet du mémoire qui lui était soumis sans s'exposer aux plaintes d'autres corps ou aux remontrances de l'État. Ces règles informelles furent le plus souvent respectées, quelles que soient l'importance du solliciteur, la question à traiter ou les tendances expansionnistes de certains académiciens ${ }^{3}$. Ce n'est qu'avec les grandes commissions du ministère Breteuil, que l'Académie transgressa certains interdits.

8 Les mémoires étaient confiés par l'Académie, lors de ses séances bihebdomadaires, à une commission constituée d'un ou de plusieurs académiciens ${ }^{4}$ dont la tâche était de valider ou non le travail présenté. Son rapport s'appuyait sur des considérations de forme (logique du plan; cohérence des assertions formulées) et de fond (reconnaissance des savoirs ; validité des expériences décrites ; intérêt du travail). Une fois rédigées sous forme d'un rapport signé et daté, les commissaires soumettaient leurs conclusions à l'Académie dans le cadre de ses travaux ordinaires. Les rapporteurs suggéraient soit le rejet, soit l'approbation du mémoire et parfois, son impression dans les recueils académiques. Enfin, toutes les commissions étaient temporaires. L'approbation de leur rapport par l'Académie mettait officiellement fin à leur existence.

9 Sur un ensemble de sources consultées ${ }^{5}$, un recensement partiel fournit un aperçu des commissions de 1760 à 1789, et permet de tirer un certain nombre de constatations ${ }^{6}$.

On note une moyenne de 170 commissions nommées $\operatorname{par}^{a^{7}}$, pour lesquelles le nombre des commissaires et la nature des travaux varient.

De 1760 à 1789 , les commissions de deux membres se taillent la part du lion ${ }^{8}$, bien qu'elles fléchissent sensiblement les neuf dernières années. Par contraste, les autres formations varient selon l'époque considérée. Les années 1760 à 1779 sont celles des 
équipes restreintes, les commissions à un membre précédant celles à trois commissaires et les commissions de quatre membres et plus atteignant péniblement les $2 \%$. Les valeurs s'inversent au cours des dernières années, les commissions de trois membres et plus devançant largement celles à un seul commissaire'. On constate enfin que les commissions comprenant cinq commissaires et plus atteignent leur plus haute valeur entre 1784 et 1788 .

En l'absence d'une classification des commissions par objet et par commissaire, il a paru intéressant d'utiliser celle réalisée pour l'académicien Jean-Sylvain Bailly ${ }^{10}$. Sur les quelque 150 commissions auxquelles il a participé en 27 ans de vie académique effective, on constate que jusque dans les années 1780 , celles à deux commissaires dominent $(63,26 \%)$ et les grandes formations sont l'exception $(5,35 \%)^{11}$. Par ailleurs, la majorité de ses commissions (85\%) traite de sujets relevant de la classe d'astronomie et par extension des classes de mathématiques. Toutefois, il n'apparaît jamais comme le spécialiste d'une question spécifique auquel l'Académie aurait recours chaque fois que le sujet serait abordé. Des mémoires portant sur des objets similaires étaient indifféremment attribués à tel ou tel académicien de la classe concernée. Cette absence (ce refus!) de spécialisation empêchait toute appropriation d'un sujet par un savant mais pouvait surtout aboutir à des rapports contradictoires entre les commissions académiques.

Il reste que $15 \%$ des commissions auxquelles Bailly fut associé se situent hors du champ de l'astronomie et des mathématiques. Cette tendance s'accentue pour atteindre $50 \%$ de son travail à partir de 1784. Parallèlement, c'est au sein de grandes formations qu'il effectue l'essentiel de ses tâches académiques.Cependant, le parcours de Bailly, classique jusqu'en 1784, s'est singularisé par son active participation aux grandes commissions de l'ère Breteuil. angles :

16 1) celles qui sont liées à une discipline. Les savants sont choisis selon leur spécialité et non par leur appartenance à une classe, ce qui autorise une certaine liberté dans leur désignation ${ }^{12}$. Puis, à partir des années 1780 , certaines classes commencent à être désignées en tant qu'ensemble ${ }^{13}$. Toutefois, la classe restant un espace restreint, on continue de lui associer des académiciens qui n'en font pas partie mais dont les compétences sont utiles à l'examen de certains mémoires ${ }^{14}$.

17 2) celles qui font appel à plusieurs disciplines. La mise en place d'équipes interdisciplinaires devient une nécessité pour aborder des thèmes de recherche de plus en plus complexes. Lavoisier l'expose clairement lors de sa demande de création d'une commission permanente des ballons ${ }^{15}:$ "Il faudrait que cette commission fût assez nombreuse pour y réunir tous les genres de sciences que l'objet comporte.» On y observe aussi un souci de parité entre les disciplines mathématique et physique.

3) celles qui se situent hors des domaines traditionnels de l'Académie. Ces commissions pénètrent dans les domaines de l'assistance publique et de la santé, de l'aménagement urbain et de la sécurité civile, sujets réservés jusque-là à d'autres experts (médecins, ingénieurs, architectes). L'Académie les avait parfois rencontrés lors de l'examen de mémoires mais n'était que rarement associée à des travaux d'importance sur ces thèmes ${ }^{16}$. Or, en 1780 , Necker demande à l'Académie de jouer un rôle d'expert et de juger en opportunité un projet relevant du domaine de la santé publique ${ }^{17}$. Cependant, 
il ne fait pas appel à ses lumières pour l'épineux problème de l'Hôtel-Dieu auquel il tente d'apporter une solution avec sa propre équipe. Il faut attendre l'installation du nouveau ministre de la Maison du Roi, le baron de Breteuil, pour que l'État renouvelle ce type de relations avec la Compagnie ${ }^{18}$. Ce mouvement rencontre l'adhésion de Condorcet et Lavoisier, qui occupent au même moment des postes clés ${ }^{19}$ et dont les velléités expansionnistes ${ }^{20}$ et de modernisation du corps ${ }^{21}$ sont connues. Enfin, l'intérêt du roi et du public pour les sciences, les nouveautés et la cause des malheureux, ne peut que servir ces ambitions.

Lors de la saisine de l'Académie par le Châtelet sur un litige entre fondeurs, Lavoisier précise dans le rapport dont il est le principal auteur, l'importance de la Compagnie et son rôle dans la société :

«Dans l'origine l'académie a été principalement instituée pour examiner toutes les inventions nouvelles qui lui seroient renvoyées de la part du Roi. Insensiblement elle est devenue un tribunal volontaire dont les particuliers réclament directement le jugement, et elle a tenu à honneur de répondre à la confiance du public.

Depuis, le travail qu'elle a entrepris pour la description des arts et métiers a augmenté encore considérablement l'étendue de sa juridiction : le parlement et $\mathrm{M}$. le Lieutenant général de police l'ont fréquemment consulté sur des objets soit d'art, soit de mécanique, on ne voit pas que dans aucune occasion elle ait cherché à éloigner les occasions de se rendre utile. [...] Ce n'est point un simple expertage qu'on lui demande. $»^{22}$

Le mode de désignation des commissaires auxquels l'Académie confiait l'examen des mémoires achève de nous renseigner sur les relations entre ce corps et l'État. Comme on l'a vu précédemment, l'objet du mémoire déterminait généralement la ou les disciplines concernées et donc par voie de conséquence les académiciens. Les ordres du jour des séances n'étant jamais improvisés, les mémoires présentés chaque semaine à l'Académie faisaient l'objet d'un tri et d'une attribution préalables vraisemblablement en accord avec les académiciens pressentis.Toutefois, comme le laisse à penser la composition des grandes commissions, le choix des commissaires n'a pas dépendu des seuls critères scientifiques. Les relations personnelles, la proximité d'intérêts avec le sujet à traiter, le rang occupé et la notoriété de chaque académicien eurent leur importance. On voit ainsi se dessiner des associations épisodiques qui surprennent et les mémoires fastidieux sont abandonnés aux jeunes adjoints. Toutefois, si dans la majorité des cas, le choix des commissaires appartenait à l'Académie, certains sujets (qualité de l'auteur; nature sulfureuse du sujet; intérêt économique, social ou de sécurité publique...) étaient soumis plus que d'autres à la censure royale. Le rôle des officiers et notamment des présidents, académiciens honoraires et grands seigneurs proches de la Cour, est à ce titre particulièrement intéressant.

21 Les grandes commissions des années 1780 évoluent donc peu à peu sous l'influence de plusieurs volontés qui se servent l'une l'autre. D'une part, des gouvernants, Necker et surtout Breteuil, soucieux d'une reprise en main de certains domaines d'intérêt public laissés depuis trop longtemps aux mains d'instances privées ou ecclésiastiques. De l'autre, des académiciens soucieux de développer le domaine de leurs propres recherches mais aussi de faire de l'Académie le moteur du monde scientifique ainsi qu'un outil performant au service d'une société dans laquelle ils veulent aussi jouer un rôle.

... moteurs de la recherche et auxiliaires du pouvoir 

celle sur le magnétisme animal créée en avril 1784 et celle des hôpitaux créée en décembre 1785. En l'espace de deux ans, ces commissions constituent des étapes majeures dans le processus d'évolution de l'institution ainsi que dans celui de ses relations au pouvoir. Celle des ballons apparaît comme une commission scientifique spécialisée à vocation permanente, qui sans représenter d'enjeu politique bénéficie du soutien du roi. Celle sur le mesmérisme, spécialisée mais temporaire, requiert de par le caractère sulfureux de son objet toute l'attention de l'État. Enfin, celle sur les hôpitaux, tout à la fois spécialisée et quasi permanente, qui marque la mise en place d'une nouvelle politique en matière d'assistance publique et par là même se trouve au cœur d'enjeux de pouvoir.

cene laps de temps l'Académie entreprit une réforme qui aboutit au règlement du 23 avril 1785. Outre la disparition des surnuméraires et des adjoints, elle permit la création de deux nouvelles classes et l'extension de deux autres ${ }^{23}$. L'Académie en étendant ses domaines de compétence et en centralisant les savoirs apparaissait plus que jamais comme la meilleure et l'unique interface entre le monde savant et le gouvernement. Dans le même temps, certains savants participaient activement à la mise en place de comités d'experts auprès des ministres ${ }^{24}$.

La commission des aérostats

24 Le 28 juin 1783, le contrôleur général des Finances, Lefèvre d'Ormesson, transmit à l'Académie pour avis, le rapport qu'il avait reçu sur l'ascension d'un ballon à air chaud effectuée par les frères Montgolfier le 4 juin. L'Académie nomma une commission de 5 membres, bientôt élargie à 8 , chargée de «constater les effets d'une machine aérostatique inventée par MM. de Montgolfier $»^{25}$ et dont le rapport approuvé par l'Académie le 23 décembre 1783 mettait officiellement fin à ses fonctions. Or, profitant de l'engouement du public, des savants et du roi lui-même pour ces machines, Lavoisier dès le 23 novembre exposait la nécessité de créer une commission permanente sur cet objet. Son argumentaire tenait en deux points :

- la nécessité pour l'Académie de poursuivre des travaux importants qui ont retenu l'attention du public et du roi : «Cette découverte a fait trop de sensation à la Cour, à la ville, à l'étranger, pour que l'académie puisse se dispenser de donner une sorte d'appareil et de publicité aux recherches dont elle doit s'occuper. »

- l'obligation de créer une commission spéciale afin de ne pas alourdir le travail ordinaire de la compagnie: "Il faut éviter que les travaux ordinaires de l'Académie, ceux qui tendent véritablement aux progrès des sciences ne soient point entièrement occupés de propositions et de discussions relatives aux machines aérostatiques. »

Fort du soutien du duc de La Rochefoucauld et de l'abbé Bossut, respectivement nommés président et directeur pour 1784, Lavoisier, lui-même nouvel officier, obtint gain de cause. L'Académie tout en dissolvant la commission des aérostats nommait sans plus de précisions des commissaires "pour les expériences sur les machines aérostatiques ». Les membres désignés furent outre Lavoisier, le duc de La Rochefoucauld, l'abbé Bossut, Leroy, Brisson, Coulomb, Berthollet et le marquis de Condorcet. Meusnier, d'abord membre associé, devint membre ordinaire dès son élection académique ${ }^{26}$. Ces commissaires représentaient une parité quasi parfaite entre les disciplines mathématiques et physiques.

Pour les mathématiques

Annales historiques de la Révolution française, 320 | avril-juin 2000 

structure de travail en équipe, spécialisée et à durée indéterminée, puisqu'elle ne dépend plus de la remise d'un rapport et que son objet se renouvelle constamment. Toutefois, elle ne bénéficie d'aucun statut qui l'identifierait officiellement.

La commission sur le magnétisme animal

41 Cette commission marque très clairement l'intrusion du politique dans les travaux académiques. La désignation des commissaires pour l'étude du magnétisme animal en avril 1784, est à ce titre très instructive. Contrairement à tous les usages, c'est le roi lui- 
même par l'intermédiaire de son ministre, le baron de Breteuil, qui désigne les commissaires pour examiner les travaux de Mesmer.

Cependant, le gouvernement ne fit pas immédiatement appel aux lumières académiques. En effet, l'examen d'une nouvelle thérapie relevant de la compétence du corps médical, le roi avait nommé une première commission composée de quatre membres de la Faculté de médecine de Paris $^{30}$. En but aux polémiques, concurrencée par la jeune Société royale de médecine, le corps vieillissant de la Faculté de médecine de Paris était en outre fortement divisé sur la question du mesmérisme. Aussi les quatre médecins désignés par le roi préférèrent s'associer le précieux concours de l'Académie des sciences dont la crédibilité n'était pas entamée. Le 2 avril 1784, le roi nommait les académiciens Poissonnier, Lavoisier, Leroy et Bailly ${ }^{31}$ :

«Le Roi a fait choix de vous, Monsieur, pour procéder, avec plusieurs autres personnes distinguées par leurs lumières et leur experience, à l'examen de la méthode ou pratique tirée des prétendues connaissances du sieur Mesmer [...] Je ne doute point que vous ne remplissiez cette commission avec le zèle et l'attention qu'elle exige. Lorsque MM. les Commissaires en auront rédigé un rapport détaillé et leur avis, j'en rendrai compte à Sa Majesté.

P.S. Ce sont, Monsieur, les médecins eux-mêmes qui ont désiré de concerter leur rapport et leur avis avec des membres de l'Académie. ${ }^{32}$

43 Le baron de Breteuil sollicitait également la participation de Franklin, «ministre plénipotentiaire des États-Unis de l'Amérique $»^{33}$ et associé étranger de l'Académie des sciences de Paris. La commission fut donc composée de cinq académiciens et de quatre médecins ${ }^{34}$.

Les médecins précisèrent-ils les académiciens avec lesquels ils désiraient collaborer? Rien ne l'indique. Toutefois, la nomination de Bory, chef d'escadre et associé libre de l'Académie, se fit le 7 mai à la demande des commissaires eux-mêmes ${ }^{35}$, ainsi que le remplacement du médecin Borie décédé, par Majault ${ }^{36}$. On constate que le baron de Breteuil était largement ouvert aux demandes et suggestions des commissaires dans la mesure où elles n'entraient pas en opposition avec les vœux du gouvernement. Tous les commissaires nommés étaient considérés à juste titre, comme des hommes sûrs qui s'empressèrent "d'entrer dans les vues de l'Administration $»^{37}$. Les correspondances entre Breteuil et Bailly, auteur du rapport de la commission, ainsi que celles échangées avec Lenoir, lieutenant de police de Paris, démontrent clairement tout l'intérêt de l'État pour cette affaire qui toucha à la sécurité publique et qui éclaboussa même la Cour.

Il est remarquable de noter que cette commission échappa totalement au contrôle de l'Académie. En effet, à aucun moment cette commission n'est évoquée dans les procèsverbaux des séances. Ce n'est que lors de la séance publique du 4 septembre 1784 au cours de laquelle Bailly lut le rapport de la commission, précédemment approuvé par le roi, que l'Académie retrouva pour la forme son pouvoir de tutelle sur la commission.

Cependant, l'Académie devait y trouver également son compte. Protectrice des sciences, elle ne pouvait rester indifférente au : « règne mal fondé des fausses opinions : les sciences qui s'acroissent par les vérités, gagnent encore à la suppresion d'une erreur $»^{38}$ et par l'objet même de sa mission, l'expert scientifique devenait, au même titre que l'État, le garant des intérêts des citoyens :

« [...] il fallait éclairer ceux qui doutaient, il fallait établir une base sur laquelle pussent venir se reposer ou l'incrédulité ou la confiance [...] une erreur est toujours un mauvais levain qui fermente et qui corrompt à la longue la masse dans laquelle elle est introduite. Mais lorsque cette erreur sort de l'empire des sciences pour se 
répandre dans la multitude, pour partager et agiter les esprits, lorsqu'elle présente un moyen trompeur de guérir à des malades qu'elle empêche de chercher d'autres secours, lorsque surtout elle influe à la fois sur le moral et sur le physique, un bon gouvernement est intéressé à la détruire. » d'État siégeant au Conseil, il avait droit de regard sur l'administration hospitalière et charitable, exerçait un contrôle sur la recherche médicale et la diffusion de nouveaux remèdes et médications. Ministre de Paris, il intervenait aussi sur les questions touchant à la sécurité et à l'urbanisme et enfin, ministre de tutelle des académies, dont il fut membre honoraire, il en devint un protecteur efficace. Par ailleurs, convaincu que l'accroissement de la prospérité de la nation dépendait en grande partie du développement et du perfectionnement des sciences et des techniques, il mena une politique interventionniste en faveur des réformes académiques et favorisa le progrès des sciences nouvelles ${ }^{43}$. Ses contemporains lui reconnurent une grande largeur de vue et de sincères préoccupations humanitaires malgré une incontestable raideur vis-à-vis 
de toute évolution politique, dont il fit notamment preuve lors des journées insurrectionnelles de juillet 1789 . qu'éclairée ", se composait de : Bailly, Darcet, Daubenton, Laplace, Lassone, Lavoisier, Leroy et Tenon. Le 13 décembre, Leroy, auteur d'un mémoire sur les hôpitaux, fut remplacé par Coulomb. Le baron de Breteuil savait qu'il pouvait compter sur ces savants pour mener à bien son entreprise. Certains étaient ou devinrent ses familiers ${ }^{44}$ et d'autres jouissaient de la confiance de la famille royale ${ }^{45}$. L'abondante correspondance de Breteuil permet de mesurer l'investissement personnel du ministre et laisse percevoir son influence lors de la désignation des commissaires. expérience acquise au cours de 42 ans de pratique médicale dans les hôpitaux de Paris et de province, et au fait des connaissances administratives et scientifiques, il rédigea de remarquables mémoires sur les hôpitaux et étudia le système hospitalier anglais en 1787 avec Coulomb. Mais la figure centrale de cette commission fut Bailly. Son rôle et les rapports qu'il rédigea lui assurèrent une solide réputation de savant et d'honnête homme ainsi que celle d'ami des malheureux. Locuteur privilégié de Breteuil, il mena notamment les négociations pour l'implantation de deux des nouveaux hôpitaux auprès de l'archevêque de Paris le 24 février 1787.

Comme il convenait pour toute commission, la remise de son rapport, le 22 novembre 1786, devait marquer sa dissolution. Mais ses conclusions appelant de nouveaux travaux d'enquête, Breteuil demanda à l'Académie le maintien en l'état de cette commission dans une lettre du 29 décembre $1786^{47}$. À l'issue de ses deux autres rapports ${ }^{48}$, elle fut chargée par le roi de coordonner la construction des nouveaux hôpitaux qu'elle prescrivait, confiant à l'Académie des sciences un nouveau rôle, mais aussi un nouveau pouvoir.

entrepris, Breteuil et Bailly proposèrent de transformer cette commission académique en comité permanent, organe semi officiel spécialisé pour toutes les questions relevant des Hôpitaux de Paris et à terme de l'ensemble des hôpitaux français ${ }^{49}$. La commission restait académique pour la forme. Faute de temps et face aux réticences, ce projet n'aboutit pas. Toutefois, la commission des hôpitaux se maintint en la forme après la chute de son protecteur. Le 20 janvier 1789, par l'entremise de M. de Villedeuil, nouveau ministre de la Maison du roi, la commission des hôpitaux se voyait confier par l'Académie le soin d'examiner 18 mémoires sur l'établissement des tueries hors de Paris et le 25 avril un projet de transporter les cimetières hors des villes. Celui qui reçut les hommages de l'Académie "pour le zèle dont il a fait preuve en faveur du progrès de la science " ${ }^{50}$ avait tracé la voie.

57 Faute de moyens, entravées par la mauvaise volonté des administrateurs des hôpitaux et privées du soutien du baron de Breteuil, les réformes proposées tournèrent court et les fonds récoltés ${ }^{51}$ atterrirent dans les caisses toujours vides de l'État. Bailly quant à lui n'abandonna pas la question des hôpitaux, et coauteur du cahier du tiers état de Paris, il exhorta au nom des malades, les États généraux à honorer les obligations sacrées.

Pour une conclusion

58 Les grandes commissions académiques, peu à peu transformées, tendirent à devenir de véritables comités d'experts consultés par le gouvernement, soucieux de s'adjoindre les compétences d'un corps fidèle, efficace et crédible. En retour, elles facilitaient la

Annales historiques de la Révolution française, 320 | avril-juin 2000 
professionnalisation d'un monde savant qui se démarquait du charlatanisme ambiant par la qualité de ses travaux et par les méthodologies mises en place. Elles lui offraient également un moyen d'assurer sa prééminence dans le monde scientifique et d'investir progressivement à la demande même du pouvoir, le domaine public.

Les savants que l'on y rencontra, tout occupés de leurs travaux scientifiques et techniques, ne furent jamais retranchés d'une société dans laquelle ils s'impliquèrent par leurs idées, leurs fonctions, leurs relations et réseaux. Investis d'une mission de guides dans les domaines scientifiques, il ne leur restait qu'un pas à franchir pour passer de la scène savante à la scène politique. Condorcet ne rêvait-il pas que les académies puissent un jour « diriger ceux qui, ne jugeant que sur l'autorité d'autrui et dociles à l'opinion populaire, n'échappent aux préjugés que lorsque le jugement des sociétés savantes leur dicte ce qu'ils doivent penser $\gg .^{52}$

Cet auxiliaire de l'État monarchique ${ }^{53}$ devint celui des assemblées parlementaires de la Révolution. Mais trop étroitement liée à la monarchie par ses origines et ses structures, l'Académie, comme ses consœurs, accompagna sa chute. Toutefois, la qualité de ses travaux et le besoin d'un élément fédérateur des sociétés scientifiques et de la recherche, motiva dès 1795 sa résurrection, avec les mêmes savants qui avaient composé l'Académie royale des sciences et qui avaient échappé au tranchant de la guillotine.

\section{NOTES}

1.Voir Charles C. Gillispie, Science and Polity in France at the End of the Old Regime, Princeton, University Press, 1980.

2.Jusqu'en 1785, l'Académie était constituée de 6 classes (astronomie, géométrie, mécanique, anatomie, chimie, botanique) chacune composée de 7 membres ( 3 pensionnaires, 2 associés, 2 adjoints) plus 1 adjoint géographe. Aux membres ordinaires s'ajoutaient les secrétaire et trésorier perpétuels ainsi que 12 honoraires, 12 associés libres et 8 associés étrangers plus un nombre variable de vétérans et de surnuméraires. 3. Condorcet et Lavoisier, par exemple. Voir notes 20 et 21.

4.Aux académiciens ordinaires auxquels incombait ce travail s'ajoutaient des associés libres et plus rarement des vétérans et des honoraires. Ces derniers intervenaient souvent comme simple caution. Des associés étrangers furent désignés lors de leur passage à Paris, comme Benjamin Franklin.

5.Archives de l'Académie des sciences : Listes des commissions données; Plumitifs ; Rapports des commissions ; Procès-verbaux des séances ; Table des procès-verbaux des Rapports de 1699 à 1793.

6.Le recensement a été fait à partir des commissions attribuées. On note qu'une commission pouvait étudier plusieurs mémoires et que certains mémoires ne firent l'objet d'aucun rapport. Par ordre de grandeur décroissant, on obtient : nbre mémoires>nbre commissions $>$ nbre rapports. 
7.On constate parfois de grands écarts dans le nombre des commissions : 202 en 1786, 120 en 1789.

8.Aux alentours de $64 \%$ de 1760 à 1779 et $47,1 \%$ de 1780 à 1789 .

9.De 1780 à 1789 , les commissions à : un commissaire $=2,6 \%$; trois $=31,2 \%$; quatre = $9,4 \%$; cinq et plus $=9,7 \%$.

10.Adjoint de la classe d'astronomie le 19 janvier 1763, associé de la classe d'astronomie le 14 juillet 1770, pensionnaire surnuméraire de la classe d'astronomie le 12 décembre 1784 et pensionnaire de la classe de physique générale le 23 avril 1785.

11.Les grandes commissions de 1760 à 1779 traitent le plus souvent de questions astronomiques et mathématiques.

12.Pour l'examen de la plate-forme astronomique présentée par le Duc de Chaulnes, les astronomes Le Monnier, Camus, Chappe, Maraldi, de Thury, Deparcieux, Jeaurat, Chabert, Bory, Bailly \& Fouchy. Rapport du 18 janvier 1766. Soit quatre membres de la classe d'astronomie sur sept auxquels s'ajoutent des académiciens d'autres classes et des associés libres.

13.Quatre classes sont concernées : dès 1780 celle d'astronomie, puis celles de chimie et de sciences naturelles à partir de 1787, enfin celle d'anatomie en 1789 .

14.Pour les essais d'or renvoyés par la Cour des monnaies, la classe de chimie plus Brisson et d'Arcet. Attribution 16 mai 1789.

15.Rapport du 23 novembre 1783. CEuvres de Lavoisier, T. III, Imprimerie de l'Empire, 1865, p.740.

16.Sur le meilleur moyen d'éclairer les rues de Paris, question soumise à concours par l'Académie en 1766 à la demande de Sartines, lieutenant général de police.

17.Mémoire de Colombier, médecin et de Moreau architecte de la Ville de Paris, pour l'établissement de nouvelles prisons dans l'emplacement occupé par les Cordeliers transmis le 26 janvier 1780 à l'Académie par Necker. Madame Necker s'empressa par l'intermédiaire de d'Alembert d'interroger Lavoisier, rapporteur de la commission, sur les résultats de l'enquête académique. Cuvres de Lavoisier, T.III, op. cit.

18.Les grandes commissions observées portent sur : les aérostats, le Magnétisme animal, les hôpitaux, les tueries, l'aménagement de berges de la Seine, la lutte contre les incendies, la description des arts et métiers, la création d'un corps d'ingénieurs et la réforme de l'Académie. Avec celle sur les prisons, on retrouve Lavoisier et Bailly 8 fois, Tillet 7 fois, Coulomb, Condorcet et Borda 5 fois.

19. Condorcet est secrétaire perpétuel en 1776 et Lavoisier directeur adjoint en 1784 et directeur en 1785.

20.Condorcet, "Quel doit être l'objet des académies », Histoire de l'académie royale des sciences pour l'année 1771.

21.Principal auteur de la réforme d'avril 1785, Lavoisier fut considéré comme l'auteur de précédents projets. Voir Éric Brian, « Lavoisier et le projet de classe de physique expérimentale à l'Académie royale des sciences (avril 1766) », dans Christiane Demeulenaere-Douyère (éd.), Il y a 200 ans Lavoisier, Londres-Paris-New York, Technique et documentation - Lavoisier, 1995, pp. 151-168.

22.Pour examiner en conséquence d'une sentence du Châtelet le cercle de cuivre fondu qui a fait élever une discussions entre MM. Bergevin et Thiry, fondeurs. Rapport manuscrit, signé et daté par Bailly et Lavoisier, pochette de séance du 5 février 1788.

23. Création de la classe de physique générale et de celle d'histoire naturelle et de minéralogie. La classe de chimie s'adjoint la métallurgie et celle de botanique l'agriculture. 
24.Lavoisier et le Comité d'agriculture créé le 16 juin 1785 avec Gravier de Vergennes, Lubert, Darcet, Poissonnier et Tillet, puis Dupont de Nemours à compter du 7 juillet suivant.

25.Les commissaires furent Lavoisier, Desmarets, Bossut, Leroy et Monge auxquels furent ajoutés Tillet, Brisson, Cadet et Condorcet tandis que Monge se retirait. 26.Meusnier fut élu adjoint géomètre le 28 janvier 1784 .

27.Recherches sur la préparation de l'hydrogène à grande échelle, travaux entrepris dès 1783. Ces travaux permirent aussi à Lavoisier de jeter les bases de la chimie nouvelle, dont la nomenclature parut avec le soutien du Baron de Breteuil en juin 1787. 28.Roger Hahn « Lavoisier et ses collaborateurs : une équipe au travail », dans C.Demeulenaere-Douyère (éd.), op.cit., pp. 55-63.

29.Un mémoire sur une balance aérostatique est attribué à une commission composée par Brisson et Monge. On note cependant une continuité dans le choix des commissaires.

30.Il s'agit de Darcet, nommé académicien le 4 avril 1784, Majault, Guillotin et Sallin. 31.Ibid.

32.Lettre du baron de Breteuil à Lavoisier du 2 avril 1784, in Correspondance Lavoisier, lettre $\mathrm{n}^{\circ} 496$, p.15 et Archives nartionales, série 01* / registre 495, p.164.

33.Ibid.

34.Les signataires du rapport académique furent Bailly, de Bory, Lavoisier, Franklin, et Leroy pour l'Académie et Darcet, Guillotin, Majault et Sallin pour la Faculté de Médecine. Poissonnier qui fut d'abord pressenti, présenta un rapport séparé au nom de la Société royale de Médecine.

35.Op. cit. $01^{*} 1 / 495$, p. 227.

36.Ibid., p. 264. Lettre du 24 mai 1784 à MM. les commissaires « S.M. a bien voulu, ainsi que vous le proposer, nommer en son lieu et place M. Majault médecin de la faculté de Paris ».

37.Exposé des Expériences qui ont été faites pour l'examen du Magnétisme Animal. Lu par M. Bailly, en son nom et au nom de Mrs Franklin, Le Roy, de Bory et Lavoisier. Histoire de l'Académie royale des sciences. Année 1784, A Paris, de l'imprimerie royale, 1787, pp. 6-15.

38. Rapport de Bailly, ibid.

39.Lettre de Breteuil à Bailly du 20 août 1784 où il indique qu'il a ordonné à $\mathrm{M}$. Duperron de tirer 2000 exemplaires du rapport in O*1495, op. cit., p. 434.

40.Voir le toujours très instructif ouvrage de Camille Bloch, L'assistance et l'État en France à la veille de la Révolution (1764-1790), Paris, Librairie Alphonse Picard et fils, 1908. 41.Constituée d'administrateurs des hôpitaux, de membres du Conseil d'État, de curés et du médecin du roi, Lassone, ses conclusions remises un an plus tard ne proposaient rien de nouveau.

42.Louis S. Greenbaum, « Jean-Sylvain Bailly, the Baron de Breteuil and the Four new hospitals of Paris », Clio Medica, vol. 8, 1973, pp. 261-284.

43. Voir supra note 27.

44.Tenon et Bailly.

45.Joseph Marie François de Lassone (1717-1788), pensionnaire vétéran en 1759, premier médecin de Louis XVI et de Marie-Antoinette et président de la Société royale de Médecine.

46.Jacques-René Tenon (1724-1816), chirurgien, professeur démonstrateur au Collège de Chirurgie, nommé pensionnaire anatomiste le 14 août 1773. 
47.Louis S. Greenbaum, op. cit.

48. Rapports des 5 septembre 1787 et 12 mai 1788, proposant la création de quatre ou cinq hôpitaux adaptés aux soins des malades et à l'hygiène, et qui seraient établis aux extrémités de Paris, là où vivait la partie la plus misérable de la population.

49.Louis S. Greenbaum, op. cit.

50.Séance du 26 juillet 1788.

51.Une souscription lancée en 1787, récolta 2,2 millions de livres. Les besoins étaient estimés à plus de 12 millions de livres et la Ville de Paris fut autorisée à contracter un emprunt de 12 millions de livres remboursables par voie de loterie.

52.0p. cit., Condorcet, Histoire de l'Académie royale des sciences, Année 1771, p. 51.

53.Voir Roger Hahn, L'anatomie d'une institution scientifique : l'académie des sciences de

Paris, 1666-1803, Paris, Éditions des archives contemporaines, 1993.

\section{RÉSUMÉS}

Création de Colbert, l'Académie royale des sciences reste intimement liée à l'État dont elle sert régulièrement les visées utilitaires. Établissement de cartes et de plans, examen de mémoires pour la délivrance des privilèges, l'Académie est tout à la fois expert, juge, enquêteur et auteur de projets scientifiques. Au cours des dernières années de l'Ancien Régime, elle est plus que jamais sollicitée par le gouvernement, qui s'appuie sur ses rapports pour soutenir ses multiples ambitions. En son sein, de grandes commissions d'enquête et d'expertise sont chargées de connaître et de rendre compte de travaux débordant le cadre de ses compétences traditionnelles. La rencontre des aspirations respectives du baron de Breteuil et de Lavoisier a incontestablement favorisé l'émergence de ces nouvelles structures de travail. L'Académie des sciences devient progressivement l'auxiliaire indispensable de l'État pour connaître des questions d'intérêt public. Parallèlement, elle impose ses orientations face aux nouveaux enjeux scientifiques et techniques. Les assemblées révolutionnaires ne s'y tromperont pas.

The Royal Academy of Sciences and problems of public interest at the end of the Ancien Régime : Commissions of Inquiry and Expertise

Created by Colbert, the Royal Academy of Sciences remained intimately connected to the state and regularly served in pursuit of its utilitarian goals. Preparing maps and plans, as well as examining memoirs in order to grant permission for publication, the Academy was at the same time expert, judge, investigator and the author of scientific projects. During the last years of the Ancien Régime, it was more than ever solicited by the government, which used the Academy's reports to support its many goals. Within the Academy, major commissions of inquiry and expertise were charged with familiarizing themselves with and reporting on work which went beyond the scope of its traditional sphere of competence. The coincidence of the Baron de Breteuil's and Lavoisier's aspirations undoubtedly favored the emergence of these new structures for the work. The Academy of Sciences progressively became an indispensable auxiliary of the state in its task of informing itself with respect to questions of public interest. In parallel, the Academy imposed its own orientation with respect to new scientific and technical issues. The Revolutionary assemblies were not to be fooled by the Academy. 
INDEX

Mots-clés : Académie des sciences, commissions, utilité publique, expertise, Breteuil

AUTEUR

PASCALE MAFARETTE-DAYRIES

Doctorante en histoire. Institut d'histoire de la Révolution française (IHRF) 